\title{
Crystal Structure of Talaromyces cellulolyticus (Formerly Known as Acremonium cellulolyticus) GH Family 11 Xylanase
}

\author{
Misumi Kataoka • Fusamichi Akita • Yuka Maeno • \\ Benchaporn Inoue • Hiroyuki Inoue • Kazuhiko Ishikawa
}

Received: 7 May 2014 / Accepted: 29 July 2014 /

Published online: 20 August 2014

(C) The Author(s) 2014. This article is published with open access at Springerlink.com

\begin{abstract}
Talaromyces cellulolyticus (formerly known as Acremonium cellulolyticus) is one of the mesophilic fungi that can produce high levels of cellulose-related enzymes and are expected to be used for the degradation of polysaccharide biomass. In silico analysis of the genome sequence of $T$. cellulolyticus detected seven open reading frames (ORFs) showing homology to xylanases from glycoside hydrolase $(\mathrm{GH})$ family 11 . The gene encoding the GH11 xylanase C (TcXylC) with the highest activity was used for overproduction and purification of the recombinant enzyme, permitting solving of the crystal structure to a resolution of $1.98 \AA$. In the asymmetric unit, two kinds of the crystal structures of the xylanase were identified. The main structure of the protein showed a $\beta$-jelly roll structure. We hypothesize that one of the two structures represents the open form and the other shows the close form. The changing of the flexible region between the two structures is presumed to induce and accelerate the enzyme reaction. The specificity of xylanase toward the branched xylan is discussed in the context of this structural data and by comparison with the other published structures of xylanases.
\end{abstract}

Keywords X-ray crystal structure $\cdot$ Biomass $\cdot$ Xylanase $\cdot$ Hemicellulose $\cdot$ Talaromyces cellulolyticus (formerly known as Acremonium cellulolyticus) $\cdot \beta$-Jelly roll

\section{Introduction}

Cellulose and xylan are linked together in plant cell walls. Xylan is one of the major structural components of plant cell wall and the second-most abundant renewable resource biomass in nature. Xylan consists of xylose moieties polymerized via a series of $\beta-1,4$-xylosidic bonds. Xylanases (endo-1,4- $\beta$-xylanases; EC 3.2.1.8) catalyze the hydrolysis of $\beta$-1,4-xylosidic bonds of xylan, constituting a class of enzymes that are critical for the degradation of hemicellulosic polysaccharides in biomass. Based on amino acid sequence similarities,

M. Kataoka $\cdot$ F. Akita $\cdot$ Y. Maeno $\cdot$ B. Inoue $\cdot$ H. Inoue $\cdot$ K. Ishikawa $(\bowtie)$ Biomass Refinery Research Center, National Institute of Advanced Industrial Science and Technology (AIST), 3-11-32, Kagamiyama, Higashihiroshima, Hiroshima 739-0046, Japan e-mail: kazu-ishikawa@aist.go.jp 
xylanases typically can be classified into either of two groups (family 10 and family 11) of glycoside hydrolases (GH; www.cazy.org/) [1-6]. The structures of GH10 and GH11 xylanases generally fold into $(\beta / \alpha)_{8}$-barrel and $\beta$-jelly roll structures, respectively. Filamentous fungi produce a wide spectrum of enzymes capable of degrading cellulose and xylan [7-9].

Talaromyces cellulolyticus (formerly known as Acremonium cellulolyticus; originally isolated by Yamanobe et al. in 1982) is a fungus displaying one of the highest known levels of cellulolytic enzyme production [10]. It was proposed that A. cellulolyticus is a new species, Talaromyces cellulolyticus, on the basis of morphology and molecular evidence [11]. Fujii et al. reported that the culture supernatant from Talaromyces cellulolyticus had a higher cellulose-specific activity and glucose yield from lignocellulosic materials than that from Trichoderma reesei [12]. Sequence analysis of the genome database of Talaromyces cellulolyticus revealed the presence of seven open reading frames (ORFs) with homology to the GH11 xylanase family [13]. Among these seven putative GH11 xylanases (Xyl11A, Xyl11B, Xyl11C, Xyl11D, Xyl11E, Xyl11F, and Xyl11G), Xyl11C (TcXylC) exhibited the highest specific activity toward xylan [13]. To our knowledge, a crystal structure has not previously been reported for a xylanase from Talaromyces cellulolyticus. In this paper, we report the cloning and overexpression of the gene encoding TcXylC, permitting us to solve the crystal structure and clarify its function.

\section{Methods}

\section{Construction of the Truncated Enzyme}

From the genome database of Talaromyces cellulolyticus, seven kinds of ORF showing the homology to GH11 xylanase $(x y l A, x y l B, x y l C, x y l D, x y l E, x y l F$, and $x y l G)$ were found [13]. According to analysis using GENETYX (www.genetyx.co.jp/), it was revealed that the gene $(x y l C)$ exhibits high homology of the xylanase from Trichoderma reesei and codes for 223 amino acid residues including intron and 17 putative signal peptides. The genome DNA of Talaromyces cellulolyticus strain CF-2612 [14] was purified by anion exchange resin column using Genomictip (QIAGEN). Two exon regions without signal sequence (sequence numbers 52-272, and 336735 (Fig. 1)) of $x y l C$ gene were amplified using genome DNA. Using these fragments, the ORF (TcXylC $\Delta$ N17) was constructed and inserted into the vector (pET-11a).

\section{Expression and Purification of TcXylC $\Delta \mathrm{N} 17$}

A truncated enzyme ( $\mathrm{TcXylC} \Delta \mathrm{N} 17)$ with 17 amino acid residues (putative signal sequence) that deleted and added Met residue at the N-terminal region of $\mathrm{TcXylC}$ was expressed and purified by the following method. The constructed expression plasmid vector described above was introduced into Escherichia coli strain BL21(DE3) for recombinant protein. Cells were grown in Luria-Bertani medium addition of $100 \mu \mathrm{g} / \mathrm{mL}$ ampicillin at $37^{\circ} \mathrm{C}$ under continuous shaking. After the optical density at $600 \mathrm{~nm}\left(\mathrm{OD}_{600}\right)$ reached 0.6 , protein expression was induced with $0.1 \mathrm{mM}$ isopropyl- $\beta$-D-thiogalactopyranoside (IPTG) for $16-\mathrm{h}$ incubation at $30{ }^{\circ} \mathrm{C}$. Cells were harvested by centrifugation and pellets were frozen at $-20{ }^{\circ} \mathrm{C}$. After freezing, pellets were resuspended in Tris buffer ( $20 \mathrm{mM}$ Tris- $\mathrm{HCl}(\mathrm{pH} 8.0)$ ), and suspension was centrifugation. Periplasmic fraction was purified by anion exchange chromatography using a HiTrap Q HP column (GE Healthcare) and a $0.0-1.0 \mathrm{M} \mathrm{NaCl}$ gradient in $20 \mathrm{mM}$ Tris- $\mathrm{HCl}(\mathrm{pH}$ 8.0). TcXylC $\Delta \mathrm{N} 17$ containing fraction was dialyzed with $20 \mathrm{mM}$ Tris- $\mathrm{HCl}(\mathrm{pH}$ 


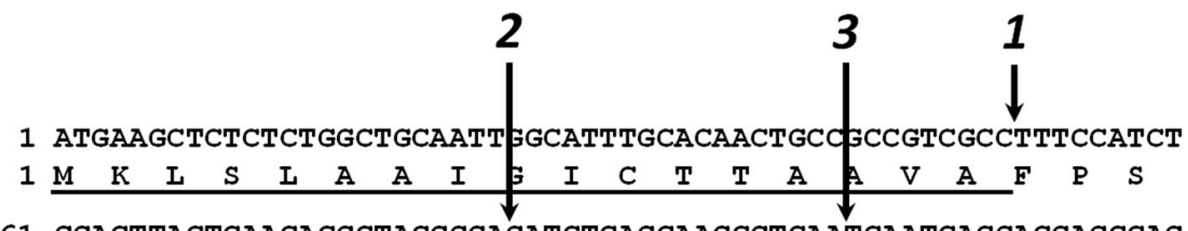

61 GGACTTACTCAACACGCTACGGGAGATCTCAGCAAGCGTCAATCAATCACGACGAGCCAG

$\begin{array}{lllllllllllllllllllll}21 & G & L & T & Q & H & A & T & G & D & L & S & K & R & Q & S & I & T & T & S & Q\end{array}$

121 ACTGGGACGAACAACGGCTACTACTACTCGTTCTGGACCAACGGCGGAGGAGAAGTCACC

$\begin{array}{lllllllllllllllllllllll}41 & T & G & T & N & N & G & Y & Y & Y & S & F & W & T & N & G & G & G & E & V & T\end{array}$

181 TACACAAATGGTGACAATGGCGAATACAGCGTGACCTGGGTCAATTGCGGTGACTTTACA

$\begin{array}{lllllllllllllllllllll}61 & Y & T & N & G & D & N & G & E & Y & S & V & T & W & V & N & C & G & D & F & T\end{array}$

241 TCTGGCAAGGGCTGGAATCCAGCTAATGCACAGTAAGTTTTCTATTTTGTTGTGTTCTAA

$\begin{array}{llllllllllllll}81 & S & G & K & G & \text { W } & \text { N } & \text { P } & \text { A } & \text { N } & \text { A } & Q\end{array}$

301 GCTTATATTTTACATACTCACATCGGAATTTGAAGGACTGTCACCTACTCTGGAGAATTT

92

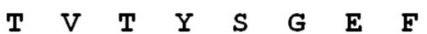

361 AATACCTCTGGAAACGCTTATCTCGCCGTTTACGGTTGGACAACTGATCCTCTTGTCGAA

$\begin{array}{lllllllllllllllllllll}100 & \text { N } & \text { T } & \text { S } & G & \text { N } & \text { A } & \text { Y } & \text { L } & \text { A } & \text { V } & \text { Y } & \text { G } & \text { W } & \text { T } & \text { T } & \text { D } & \text { P } & \text { L } & \text { V } & \text { E }\end{array}$

421 TACTACATCCTGGAGTCCTACGGTACATATAACCCATCATCTGGCCTTACATTACTTGGC

$\begin{array}{lllllllllllllllllllll}120 & Y & Y & I & \text { L } & E & S & Y & G & \text { T } & \text { Y } & \text { N } & \text { P } & \text { S } & \text { S } & \text { G } & \text { L } & \text { T } & \text { I } & \text { L } & G\end{array}$

481 CAGGTTACTAGCGATGGTGGTACGTACGATATCTACTCAACACAGCGTGTCGACCAACCC

$\begin{array}{llllllllllllllllllllll}140 & Q & V & \text { T } & \text { S } & \text { D } & G & G & \text { T } & \text { Y } & \text { D } & \text { I } & \text { Y } & \text { S } & \text { T } & Q & R & \text { V } & \text { D } & Q & P\end{array}$

541 TCCATCGAGGGAACTTCCACCTTCAATCAGTACTGGTCGGTTCGCACAGAGAAGCGAGTC $\begin{array}{lllllllllllllllllllll}160 & S & I & E & G & T & S & T & F & N & Q & Y & W & S & V & R & T & E & K & R & V\end{array}$

601 GGCGGAACTGTCACCACGGCCAACCACTTTGCAGCATGGAAGGCACTTGGACTTGAAATG $\begin{array}{lllllllllllllllllllll}180 & G & G & T & V & T & T & A & N & \text { H } & \text { F } & \text { A } & \text { A } & \text { W } & \text { K } & \text { A } & \text { I } & \text { G } & \text { I } & \text { E } & \text { M }\end{array}$

661 GGTACTTATAACTATATGATTGTGTCTACAGAAGGCTACGAGAGCAGTGGTTCTAGTACC $\begin{array}{lllllllllllllllllllllll}200 & G & T & Y & N & Y & M & I & V & S & T & E & G & Y & E & S & S & G & S & S & T\end{array}$

721 ATCACCGTGTCCTAG

220 I T V S *

Fig. 1 Deduced amino acid sequence for TcXylC protein. The putative signal peptide is underlined. The intron sequence is identified by the red color. The adenine residue (blue) was substituted for cytosine residue (silent mutation) because of the gene construction. The arrows 1, 2, and 3 show the N-termini of TcXylC $\Delta \mathrm{N} 17$, cleaved $\mathrm{TcXylC} \Delta \mathrm{N} 17$, and TcXylC $\Delta \mathrm{N} 34$, respectively (color figure online)

8.0) and concentrated using Amicon Ultra-15 (Millipore). The molecular weight and purity of the protein were checked by sodium dodecyl sulfate polyacrylamide gel electrophoresis (SDSPAGE). The protein concentration of TcXylC $\Delta \mathrm{N} 17$ was determined from UV absorbance at $280 \mathrm{~nm}\left(\mathrm{~A}_{280}\right)$, based on an extinction coefficient of 57,180 calculated from the protein sequence [15].

Molecular Weight Determination and N-Terminal Amino Acid Sequencing

The molecular weights of purified enzymes were analyzed by matrix-assisted laser desorptionionization/time-of-flight mass spectrometry (MALDI-TOF MS). The N-terminal amino acid 
sequences were analyzed by Edman degradation. Both analyses were carried out at APRO Science Inc. (Tokushima, Japan).

\section{Thermal Shift Assay}

The protein thermal shift assay (TSA) [16] was performed using real-time PCR and monitored the protein denaturation using sensitive dye, SYPRO Orange dye. The purified protein was adjusted to the concentration of $0.5 \mathrm{mg} / \mathrm{mL}$ in $20 \mathrm{mM}$ sodium acetate buffer ( $\mathrm{pH} 5.5$ ). Twenty microliters of purified protein was mixed with $1 \mu \mathrm{L}$ of SYPRO Orange dye. The fluorescence data was plotted by the real-time PCR instrument throughout the temperature range from 25 to $85^{\circ} \mathrm{C}$; a melt curve was generated, and the midpoint or $T_{\mathrm{m}}$ of the resulting curve was taken as a reference of the thermal stability of a protein of interest.

\section{Crystallization}

The purified TcXylC $\Delta \mathrm{N} 17$ was concentrated to $10 \mathrm{mg} / \mathrm{mL}$ and then dialyzed against $20 \mathrm{mM}$ Tris- $\mathrm{HCl}(\mathrm{pH} 8.0)$. The crystals of the TcXylC $\Delta \mathrm{N} 17$ were prepared and grown using a reservoir solution that consisted of $0.1 \mathrm{M}$ Bis-Tris (2-[bis(2-hydroxyethyl)amino]-

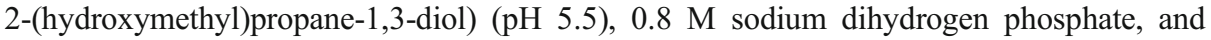
$0.8 \mathrm{M}$ potassium dihydrogen phosphate at $25^{\circ} \mathrm{C}$ by the hanging-drop vapor-diffusion method with the drops composed of equal volumes $(1.0 \mu \mathrm{L})$ of the protein and reservoir solutions equilibrated against $0.4 \mathrm{~mL}$ of reservoir solution.

\section{X-ray Data Collection}

The selected crystals were placed in the cryoprotectant solution that consisted of $33 \%(\mathrm{v} /$ v) ethylene glycol, $0.67 \mathrm{M}$ Bis-Tris ( $\mathrm{pH} 5.5), 0.53 \mathrm{M}$ sodium dihydrogen phosphate, and $0.53 \mathrm{M}$ potassium dihydrogen phosphate. The soaked crystal was collected with a cryoloop and flash-cooled under cryostream of nitrogen gas at $-173{ }^{\circ} \mathrm{C}$. X-ray diffraction data experiment was carried out at the SPring-8 BL44XU in Hyogo, Japan. The dataset was collected at a wavelength of $0.9 \AA$ using a Rayonix MX225HE detector at the beamline. The distance from the crystal to the detector was $230 \mathrm{~mm}$. The crystal was rotated at $200^{\circ}$ with an oscillation angle of $0.5^{\circ}$ per frame. The data collected from diffraction measurements were indexed, integrated, and scaled with the programs in the $H K L-2000$ software package [17].

Structure Solution and Refinement

TcXylC $\Delta \mathrm{N} 17$ structure was solved by molecular replacement with MOLREP [18] in the CCP4 package using as a search model the structure of XYNII from Trichoderma reesei (TrXyl) (Protein Data Bank (PDB; www.pdb.org/) ID 1XYP) [1] which shows $61 \%$ sequence identity with $\mathrm{TcX} X \mathrm{lC} \Delta \mathrm{N} 17$. The resulting electron density maps were used to refine and build a model of TcXylC $\Delta$ N17. Structure model-building was performed with Coot [19]. The structure was refined using REFMAC5 [20]. Water molecules were introduced at peaks over 3.0 root-mean-square deviation (RMSD) in the difference Fourier map fulfilling reasonable interactions with the protein model. Ramachandran plot of the final structure was validated using ProCheck [21]. Molecular graphics images were generated using PyMOL (www.pymol.org/). Processing parameters are presented in Table 1. 
Table 1 Data collection and refinement statistics of the structure of TcXylC $\Delta \mathrm{N} 17$

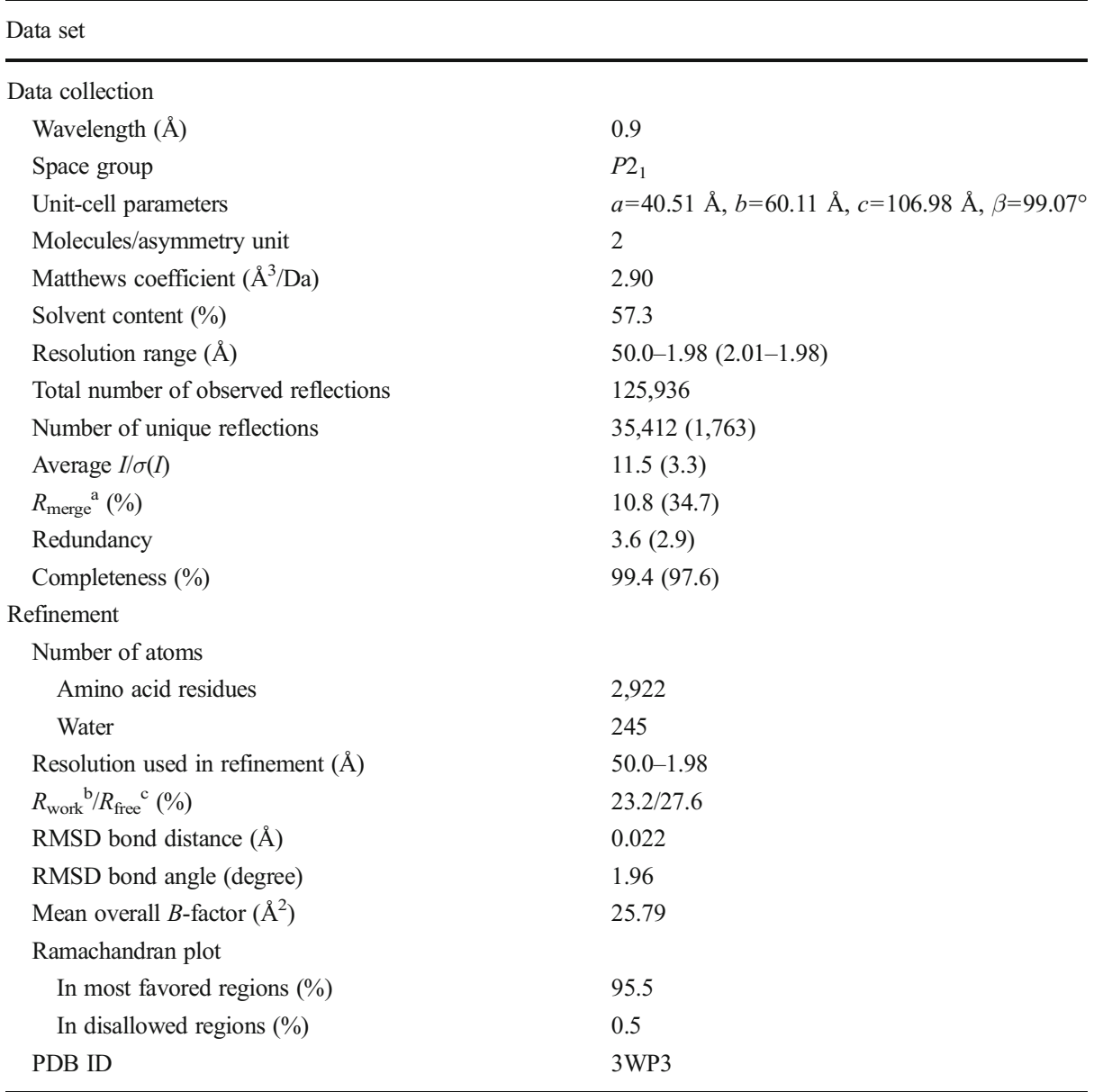

${ }^{\text {a }} R_{\text {merge }}=\Sigma_{h k l} \Sigma_{i}\left|I_{i}(h k l)-<I(h k l)>\right| / \Sigma_{h k l} \Sigma_{i} I_{i}(h k l)$, where $I_{i}(h k l)$ is the intensity of the $i$ th measurement of reflection $h k l$, including symmetry-related reflections, and $<I(h k l)>$ is their average

${ }^{\mathrm{b}} R_{\text {work }}=\Sigma_{h} \Sigma_{i}|| F_{\mathrm{o}}|-| F_{c}|| / \Sigma\left|F_{\mathrm{o}}\right|$

${ }^{\mathrm{c}} R_{\text {free }}$ is $R_{\text {work }}$ for approximately $5 \%$ of the reflections that were excluded from the refinement

\section{Results and Discussion}

\section{Preparation of the Recombinant Enzyme}

According to the analysis using GENETYX (www.genetyx.co.jp/), the putative TcXylC gene contains the intron and signal peptides (Fig. 1). An ORF encoding the putative mature enzyme (designated TcXylC $\Delta \mathrm{N} 17$ ), lacking the intron and signal regions, was constructed using PCR and inserted into the pET-11a expression vector. The recombinant enzyme was overproduced in E. coli using the pET system. The recombinant enzyme was prepared and purified as described in "Methods" section. The molecular weight of the purified TcXylC $\Delta$ N17 was determined to be about $20 \mathrm{kDa}$ by SDS-PAGE (Fig. 2). It was observed that the purified TcXylC $\Delta \mathrm{N} 17$ was cleaved to yield a smaller molecular weight 


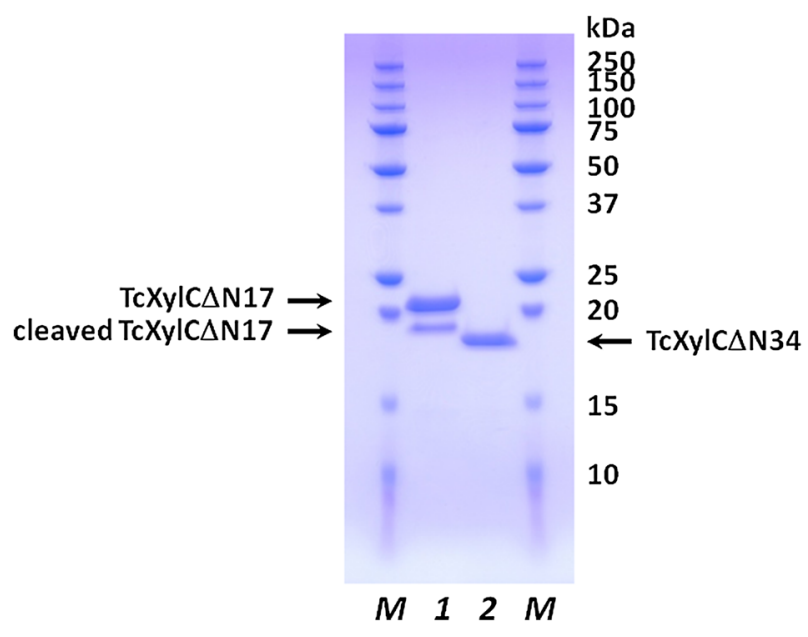

Fig. 2 SDS-PAGE analysis of two purified TcXylC enzymes. Lanes designated 1 and 2 correspond to purified TcXylC $\Delta \mathrm{N} 17$ (not fresh) and TcXylC $\Delta \mathrm{N} 34$, respectively. Note that the TcXylC $\Delta \mathrm{N} 17$ preparation includes a shortened (cleaved) product. Molecular weight standards (lane $M$ )

species (smaller than $20 \mathrm{kDa}$; designated as cleaved TcXylC $\Delta \mathrm{N} 17$ ) over time. The 34 amino acid residues of $\mathrm{N}$-terminus of the protein do not seem to be folded from the structural analysis of the xylanase (described below). Thereby, we constructed the enzyme that deleted 34 amino acid residues of $\mathrm{N}$-terminus (TcXylC $\Delta \mathrm{N} 34$ ) (Fig. 1). The overproduction and purification of TcXylC $\Delta \mathrm{N} 34$ provided yield similar to those seen for TcXylC $\Delta$ N17. SDS-PAGE analysis of TcXylC $\Delta$ N34 revealed a stable single band, as shown in Fig. 2. Based on the amino acid sequences, the calculated molecular masses of TcXylC $\Delta \mathrm{N} 17$ and TcXylC $\Delta \mathrm{N} 34$ were predicted to be $22,485.21$ and 20,660.20, respectively. In SDS-PAGE analysis, the cleaved TcXylC $\Delta \mathrm{N} 17$ ran slightly larger than TcXylC $\Delta \mathrm{N} 34$ (Fig. 2). The detailed molecular masses of the two species were determined by mass spectrometry. The results of MALDI-TOF MS analysis revealed that the total masses were 22,501.66 (TcXylC $\Delta \mathrm{N} 17), 21,231.38$ (cleaved TcXylC $\Delta \mathrm{N} 17$ ), and 20,525. 83 (TcXylC $\Delta \mathrm{N} 34$ ). To determine the cleavage sites, we examined the $\mathrm{N}$-terminal sequences of each of the proteins. These analyses showed that the N-terminal five amino acid residues of the enzymes were Met-Phe-Pro-Ser-Gly (TcXylC $\Delta$ N17), Asp-Leu-SerLys-Arg (cleaved TcXylC $\Delta$ N17), and Ser-Ile-Thr-Thr-Ser (TcXylC $\Delta$ N34), demonstrating that TcXylC $\Delta$ N17 was cleaved between Gly28 and Asp29 (Fig. 1) following overproduction in the E. coli expression system. Enzyme assays revealed that the three recombinant enzyme preparations exhibited similar specific activities for xylanase.

Thermostability of TcXylC

Thermostability of TcXylC was analyzed by TSA [16]. The result of TSA of the recombinant enzymes using the real-time PCR instrument is shown in Fig. 3. The $T_{\mathrm{m}}$ values of the TSA melting curve of purified fresh TcXylC $\Delta \mathrm{N} 17$ (not cleaved) and TcXylC $\Delta \mathrm{N} 34$ were 57.5 and $58.0^{\circ} \mathrm{C}$, respectively. The similarity of the $T_{\mathrm{m}}$ values indicates that the cleavage of $\mathrm{N}$-terminus (17 amino acid residues from $\mathrm{N}$-terminus of TcXylC $\Delta \mathrm{N} 17$ ) was unrelated to protein folding or the thermostability of the enzymes. 


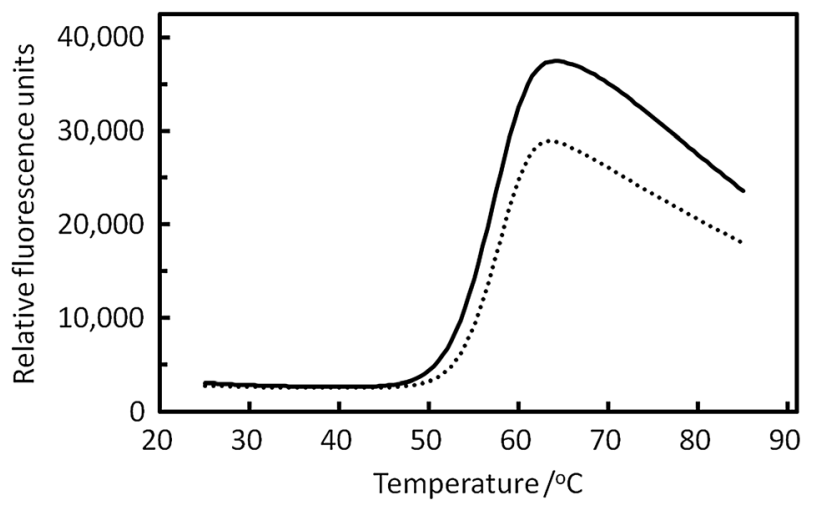

Fig. 3 Thermal stability assay of TcXylC proteins. The thermal shifts are indicated for fresh purified TcXylC $\Delta \mathrm{N} 17$ (solid line) and purified TcXylC $\Delta \mathrm{N} 34$ (dotted line)

\section{Structural Determination}

Crystal of the recombinant xylanase (TcXylC $\Delta$ N17) was prepared by the hanging-drop vapor-diffusion method. After optimization of the crystallization conditions, crystals of average size $0.1 \mathrm{~mm} \times 0.2 \mathrm{~mm} \times 0.02 \mathrm{~mm}$ (Fig. 4) were obtained within 3 days at $25{ }^{\circ} \mathrm{C}$ using a reservoir solution consisting of $0.1 \mathrm{M}$ Bis-Tris ( $\mathrm{pH}$ 5.5), $0.8 \mathrm{M}$ sodium dihydrogen phosphate, and $0.8 \mathrm{M}$ potassium dihydrogen phosphate. The statistics of data collection and refinement are summarized in Table 1. Diffraction data were collected to a resolution of $1.98 \AA$. The crystal belonged to a space group $P 2_{1}$ with unit-cell parameters $a=40.51 \AA$, $b=60.11 \AA, c=106.98 \AA, \beta=99.07^{\circ}$. The TcXylC $\Delta \mathrm{N} 17$ structure was solved by molecular replacement with $M O L R E P$ [18] in the CCP4 package using as a search model the structure of TrXyl (PDB ID 1XYP) [1]. In the asymmetric unit, two molecules of TcXylC $\Delta \mathrm{N} 17$ were observed. The presence of two enzyme molecules in the asymmetric unit gives a crystal volume per protein mass $\left(V_{\mathrm{M}}\right)$ of $2.90 \AA^{3} / \mathrm{Da}$ and a solvent content of $57.3 \%(v / v)$ [22]. After the refinement, the $R$ factors were estimated to be $R_{\text {work }}=23.2 \%$ and $R_{\text {free }}=27.6 \%$.

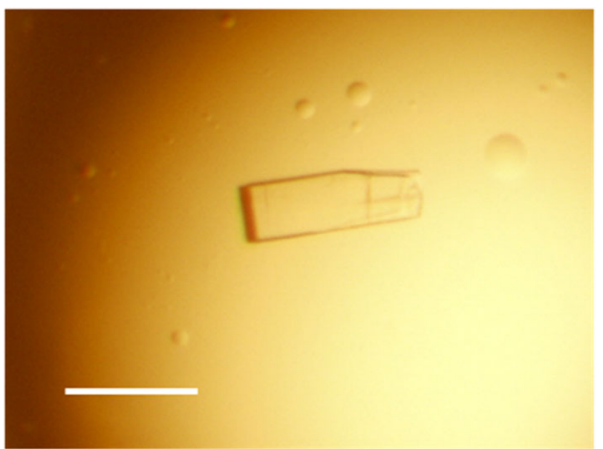

Fig. 4 A photograph of the TcXylC $\Delta \mathrm{N} 17$ crystals. The scale bar corresponds to $0.2 \mathrm{~mm}$ 
Overall Structure of TcXylC

The model of TcXylC $\Delta \mathrm{N} 17$ was able to be built from Gln34 to Ser223. The structure of the peptide sequence from N-terminus to Arg33 was not observed. The disordered region (from Met1 to Arg33) seems to be the signal sequence of the xylanase [13]. The overall structure of TcXylC $\Delta \mathrm{N} 17$ shows a $\beta$-jelly roll fold, as seen in other GH11 family xylanases (Fig. 5) [1-6]. TcXylC $\Delta \mathrm{N} 17$ consists of $14 \beta$-sheets (labeled A2 to A6 and B1 to B9) and one $\alpha$-helix (Figs. 5a and 6). The $\beta$-sheets were designated as A2 (residues 58-65), A3 (residues 68-74), A4 (residues 216-223), A5 (residues 92-101), A6 (residues 181-184), B1 (residues 40-44), B2 (residues 47-53), B3 (residues 78-83), B4 (residues 201-212), B5 (residues 104-114), B6

a

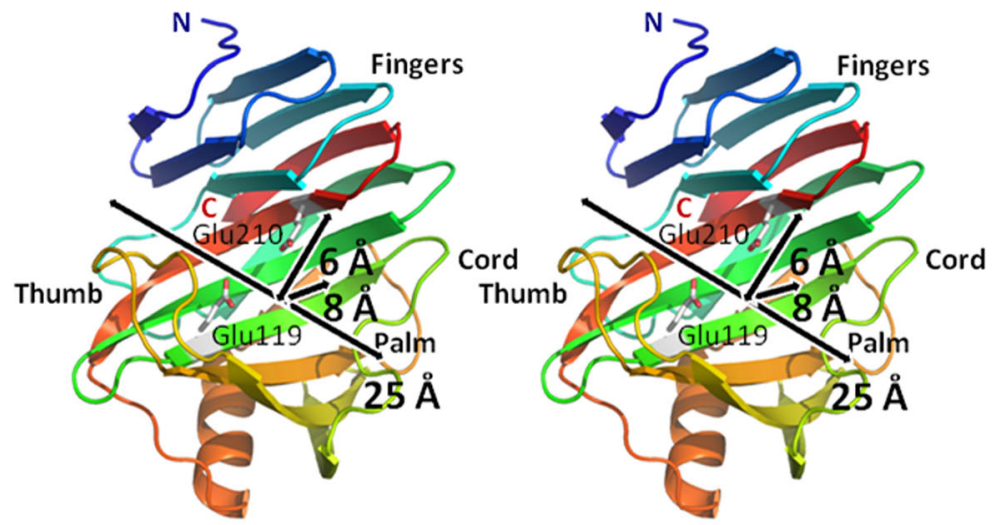

b
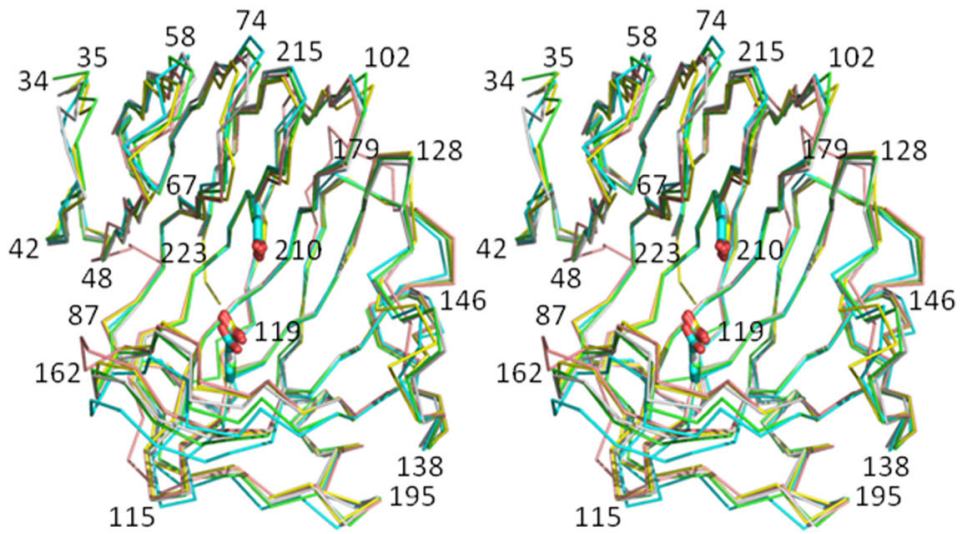

Fig. 5 Overall crystal structure of TcXylC $\Delta \mathrm{N} 17$ drawn with wall-eyed stereo view. a Ribbon model viewed from front is color-graduated according to residue number from $\mathrm{N}$-terminus with blue to C-terminus with red. The arrows show size of the active site cleft. Length, width, and depth are 25,6 , and $8 \AA$, respectively. Two of the active site residues (Glu119 and Glu210) are shown with bond model. b The model of TcXylC $\Delta$ N17 structure is superimposed with other GH family 11 xylanase structures. The distinct xylanase structures are identified by color: green (TcXylC $\Delta \mathrm{N} 17)$, cyan (TrXyl), gray (CtXyl), yellow (NpXyl), and magenta (BcXyl). Selected residues of TcXylC $\Delta \mathrm{N} 17$ are indicated by number (color figure online) 


TeXylCDN17
TrXyl
CtXyl
NpXyl

TCXY1CDN17

TrXyl

ctxyl

NpXyl

TCXYlCDN17 110

Trxyl

ctxyl

NpXyl

TCXYlCDN17 159

TrXyl

126

ctxyl

NpXyl 76 77 100
18

1

1

1

mis.

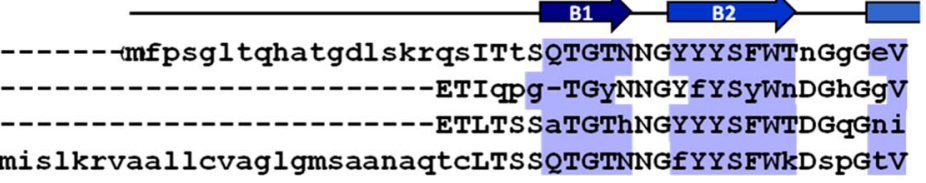

$A 2>A 3$

1 TYTNGdnGEYSVTWVNCGdFtSGKGWNP-anaqtVTYSGEFNtSGNaYLA 26 TYTNGpGGQfSVNWSNSGNEVGGKGWQPGTkNkVINISGSYNPNGNSYIS 27 rFnLESGGQYSVTWSGNGNWVGGKGWNPGTdNRVINY TadYIPNGNSYLA 51 nFCLqSGGrYtsNWSGInNWVGGKGWQtGSr-RnITYSGSFNSPGNgYLA

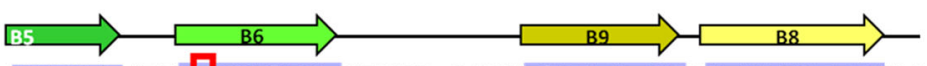

VYGWTTAPLVEYYIIESYGTYNP-SSGITILGGVTSDGGTYDIYSTQRVd VYGWSRNPLIEYYIVENFGTYNP-STGATKLGEVTSDGSVYDIYRTQRVN VYGWTRNPLIEYYVVESFGTYAP-STGATIMGSVTEDGGTYNIYRTQRVN 1 YGWTTNPLVEYYVVdSWGSWrPpgSdgTfLGtVnSDGGTYDIYRAQRVN

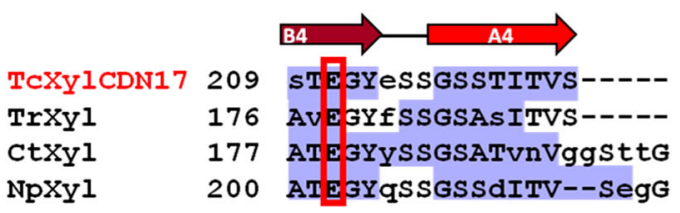

Fig. 6 Structure-based sequence alignment of TcXylC $\Delta \mathrm{N} 17$ with TrXyl, CtXyl, and NpXyl. Residues that show structural similarity are capitalized. The catalytic residues are indicated with red box. The $\beta$-sheets are colored blue boxes. The $\beta$-sheets in the TcXylC $\Delta \mathrm{N} 17$ structure are labeled $A$ and $B$. Respective strands are labeled $(A 2-$ $A 6$ or $B 1-B 9$ ) according to their positions in the two $\beta$-sheets. The $\alpha$-helix is colored yellow boxes (color figure online)

(residues 118-126), B7 (residues 167-174), B8 (residues 146-155), and B9 (residues 136144). The $\alpha$-helix corresponds to residues $185-195$. The shape of the overall GH11 family xylanase structure has been described as a "right hand" $[1,5]$. The long loop between B7 and B8 forms a "thumb." The $\beta$-sheets A and B form "fingers." The $\beta$-sheet B and the $\alpha$-helix form a "palm." By analogy to other GH11 family xylanases, it was clarified that two active site residues, Glu119 and Glu210, are located in this region. The loop between B6 and B9 forms a "cord." The structurally determined N-terminus of TcXylC $\Delta \mathrm{N} 17$ (from Gln34) corresponds to the "little finger" of fingers region, and C-terminus is located at A4, between A3 and A5. The main chain structure of TcXylC $\Delta \mathrm{N} 17$ shows high similarity with the other GH11 xylanases for which structures have been solved, including TrXyl [1], xylanase from Chaetomium thermophilum (CtXyl) [2], xylanase 11A from Neocallimastix patriciarum (NpXyl) [3], and xylanase from Bacillus circulans (BcXyl) [4] (Fig. 5b). According to sequence alignment, TcXylC $\Delta$ N17 exhibits high homologies of primary and secondary structures with these previously described xylanases (Fig. 6). Comparisons among the tertiary structures are expected to provide clues to the function of enzymes, such as the manner of substrate binding. 
Active Site Cleft

The active site cleft is approximately $25 \AA$ long, $6 \AA$ wide, and $8 \AA$ deep in close form (Fig. 5a). The two catalytic residues, nucleophile Glu119 in $\beta$-sheet B6 and proton donor Glu210 in $\beta$-sheet B4, are located in this cleft. As noted above, two individual molecules exist in the asymmetric unit and are designated here as $A_{\text {close }}$ and $B_{\text {open }}$ in Fig. 7a. The RMSDs of the C $\alpha$ atoms between $A_{\text {close }}$ and $B_{\text {open }}$ were estimated to be $0.55 \AA$, and differences in conformational regions were observed between $A_{\text {close }}$ and $B_{\text {open. }}$. The thumb in $A_{\text {close }}$ represents the close form (closed active site cleft), while the thumb in $B_{\text {open }}$ represents the open form (open active site cleft). The RMSD value of the C $\alpha$ atoms for the thumbs between $A_{\text {close }}$ and $B_{\text {open }}$ is $0.78 \AA$. According to superposition of fingers, the thumb of $A_{\text {close }}$ inclines at $3.2^{\circ}$ and close to fingers. Furthermore, the side chain of Trp52 inclines at $6.2^{\circ}$, and the main chain of Pro159 draws apart by $1.2 \AA$ (Fig. 7b). In the active site of $A_{\text {close }}$, the distance between Trp52 and Pro159, forming a tunnel, is $1.9 \AA$. In the cleft of $A_{\text {close }}, 10$ water molecules were observed, and a network of 31 hydrogen bonds were formed between the water molecules and 12 amino acid residues (Ser49, Asp78, Thr80, Tyr110, Trp112, Glu119, Tyr121, Arg155, Pro159, Tyr204, Ser208, and Glu210) (Fig. 7c). In contrast, the cleft of $B_{\text {open }}$ included 7 water molecules and a network of 21 hydrogen bonds formed between the water molecules and 14 amino acid residues (Ser49, Thr80, Asn104, Tyr110, Trp112, Glu119, Tyr121, Pro131, Arg155, Gln169, Trp171, Tyr204, Ser208, and Glu210) (Fig. 7c). Because the cross-sectional hydrogen bond networks are formed in the active site cleft, thumb gets close to finger that is formation of the tunnel. In the cleft of $A_{\text {close }}, 10$ water molecules observed seem to mimic the substrate analog. On the other hand, in $B_{\text {open, }}$ the region from $\alpha$-helix to B4 (residues 185-204) inclined at 5.4 $4^{\circ}$ and the loop between B5 and B6 (residues 114-118) and cord wrapping thumb (residues 131-143) were spread at $1.4^{\circ}$ and $2.8^{\circ}$, respectively, to outside of the enzyme by comparison with $A_{\text {close }}$ (Fig. 7d). The average values of the overall $B$-factors in $A_{\text {close }}$ and $B_{\text {open }}$ were 20.9 and $29.3 \AA^{2}$, respectively. The value of the $B$-factor in the thumb in $B_{\text {open }}$ was $54.0 \AA^{2}$. In $B_{\text {open, }}$, the values of the $B$-factor of the region from the finger to A2 (residues 34-65), the loop between B5 and B6 (residues 114-118), cord (residues 131-143), and the region from the $\alpha$-helix to B4 (residues 185-204) are $31.8 \AA^{2}$ (RMSD $0.73 \AA$ ), $42.3 \AA^{2}$ (RMSD $0.24 \AA$ ), $42.3 \AA^{2}$ (RMSD $0.68 \AA$ ), and $41.5 \AA^{2}$ (RMSD $0.35 \AA$ ), respectively (Fig. 7e). Because the values of the $B$-factors of the finger region in $A_{\text {close }}$ and thumb region in $B_{\text {open }}$ exhibit high values (Fig. 7d, e), these regions are able to migrate, providing flexibility to the individual structures. These results suggest the following properties. For forming the enzyme-substrate complex, the flexible thumb effectively binds to or incorporates the substrate in $B_{\text {open. }}$. On the other hand, the flexing of the finger region in $A_{\text {close }}$ facilitates the release of product from the enzyme-substrate complex. The changing of the flexible region between forms $A_{\text {close }}$ and $B_{\text {open }}$ is presumed to induce and accelerate the enzyme reaction.

\section{Substrate Binding}

The active site conformation of TcXylC $\Delta \mathrm{N} 17$ is highly homologous to that of $\operatorname{TrXyl}[1]$ (sequence identity $61 \%$, PDB ID 1XYP, Z-score 33.7), CtXyl [2] (sequence identity $59 \%$, PDB ID 1XNK, Z-score 34.8), NpXyl [3] (sequence identity $58 \%$, PDB ID 2VGD, Z-score 34.3), and BcXyl [4] (sequence identity $51 \%$, PDB ID 1XNB, Z-score 29.1). In TrXyl, the active site cleft of TrXyl has five xylose residue binding sites (subsites), from subsite -2 to +3 . According to superposition of TcXylC $\Delta \mathrm{N} 17$ and $\mathrm{TrXyl}$, it is estimated that the five xylose binding sites in TcXylC $\Delta$ N17 are constituted as follows: subsite -3 (Ile161, Glu162, Gly163), subsite -2 (Ser49, Trp52, Tyr110, Trp112, Pro159, Ser160, Tyr204), subsite -1 (Asp78, Phe79, Thr80, Tyr110, Glu119, Tyr121, Arg155, Glu210), subsite +1 (Asp78, Tyr106, Tyr121, Arg155, Gln169, Trp171, Glu210), subsite +2 (Asn104, Tyr129, Tyr212), and subsite +3 
a

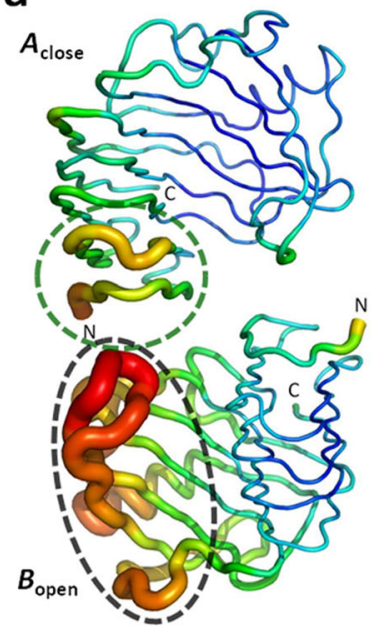

b

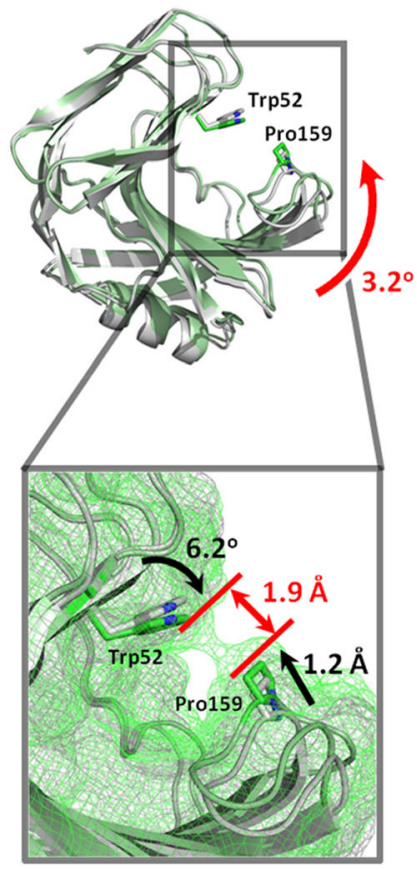

C close form $\left(A_{\text {close }}\right)$

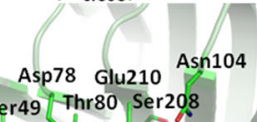

$\operatorname{Trp52} 2$ t a....... Pro13 $\longrightarrow 2$ Tyr110 Pro159 Tyr204 Tyr121 7 ? Q Trp112 $\operatorname{Arg} 155 \quad$ Glu119 Tri open form ( $\left.B_{\text {open }}\right)$
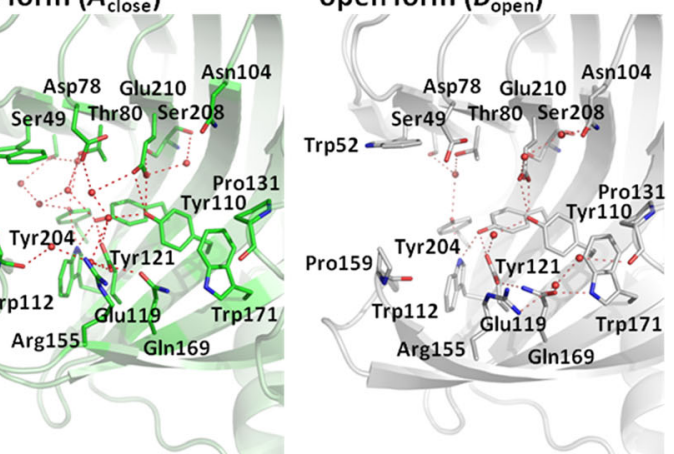

d

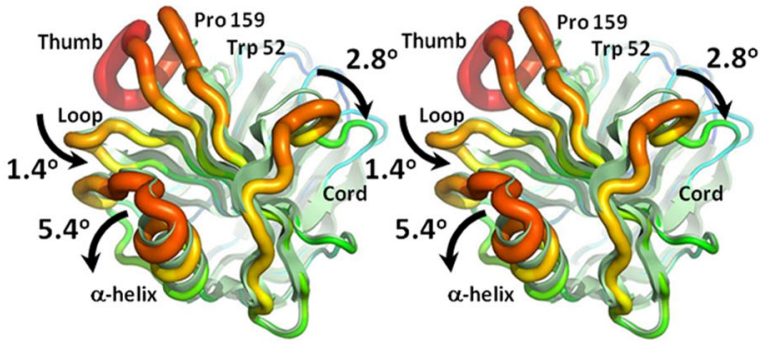

e

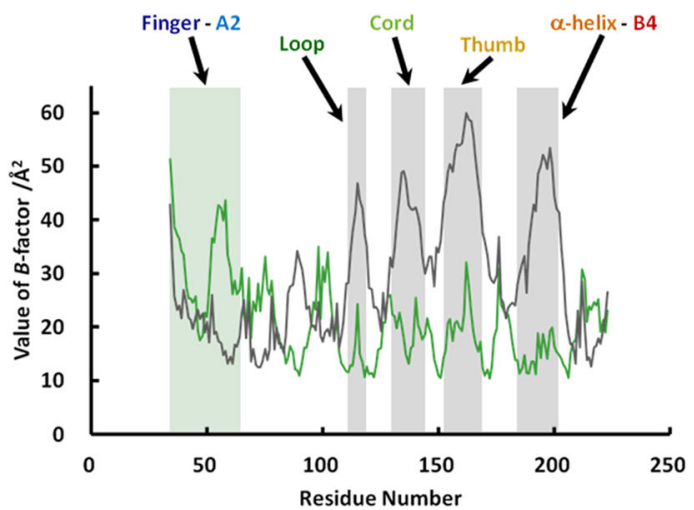

Fig. 7 Structural comparison of $A_{\text {close }}$ and $B_{\text {open }}$ crystal forms. a The $B$-factor of amino acid residues of TcXylC $\Delta$ N17. Red and blue ribbons indicate high and low $B$-factor amino acid residues, respectively. b Image showing superposition of $A_{\text {close }}$ and $B_{\text {open }}$ crystal forms. Surfaces of both subunits are drawn by mesh. c Hydrogen bond networks in the active site cleft. $A_{\text {close }}$ and $B_{\text {open }}$ are identified by color: green $\left(A_{\text {close }}\right)$ and gray $\left(B_{\text {open }}\right)$. d Wall-eyed stereo views of the structural comparison of $A_{\text {close }}$ and $B_{\text {open }}$. The structures are viewed from the bottom side. $A_{\text {close }}$ and $B_{\text {open }}$ are drawn by cartoon and ribbon models, respectively. In $B_{\text {open }}$, red and blue ribbons show high and low $B$-factor amino acid residues, respectively. e $A_{\text {close }}$ and $B_{\text {open }}$ are identified by color: green $\left(A_{\text {close }}\right)$ and black $\left(B_{\text {open }}\right)$ (color figure online) 
(Tyr129). Superposition of TcXylC $\Delta \mathrm{N} 17$ and substrate analogs complexed in CtXyl and $\mathrm{NpXyl}$ is shown in Fig. 8a. The substrate analog for CtXyl covers the subsites from -3 to -1 in TcXylC $\Delta \mathrm{N} 17$. The substrate analog for NpXyl covers the subsites from -3 to -2 and from + 1 to +3 in TcXylC $\Delta \mathrm{N} 17$. Focusing on the active site cleft of TcXylC $\Delta \mathrm{N} 17$, it is estimated that the large space opens from subsite -3 toward the nonreducing end and from subsite +2 toward the reducing end. According to the superposition, TcXylC $\Delta \mathrm{N} 17$ has enough space to accommodate some branched sugar chain substrates. On the other hand, BcXyl has a large "hump" in front of the active site (Fig. 8b). The hump in BcXyl is approximately $7.2 \AA$ wide and $2.8 \AA$ deep. In BcXyl, the main chain of the thumb, including bulky residues (Asp121 and Arg122), is

\section{a}
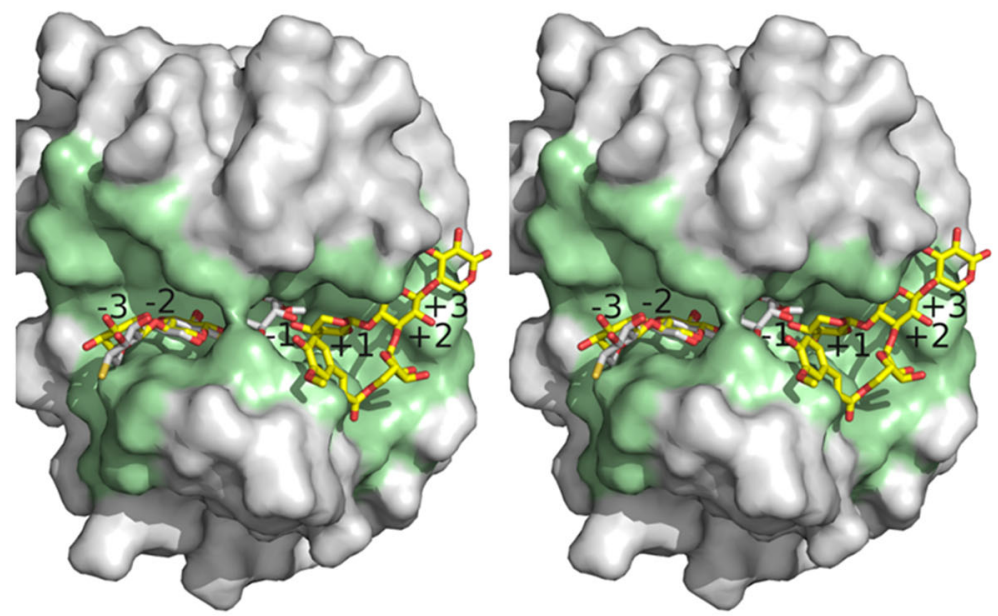

b
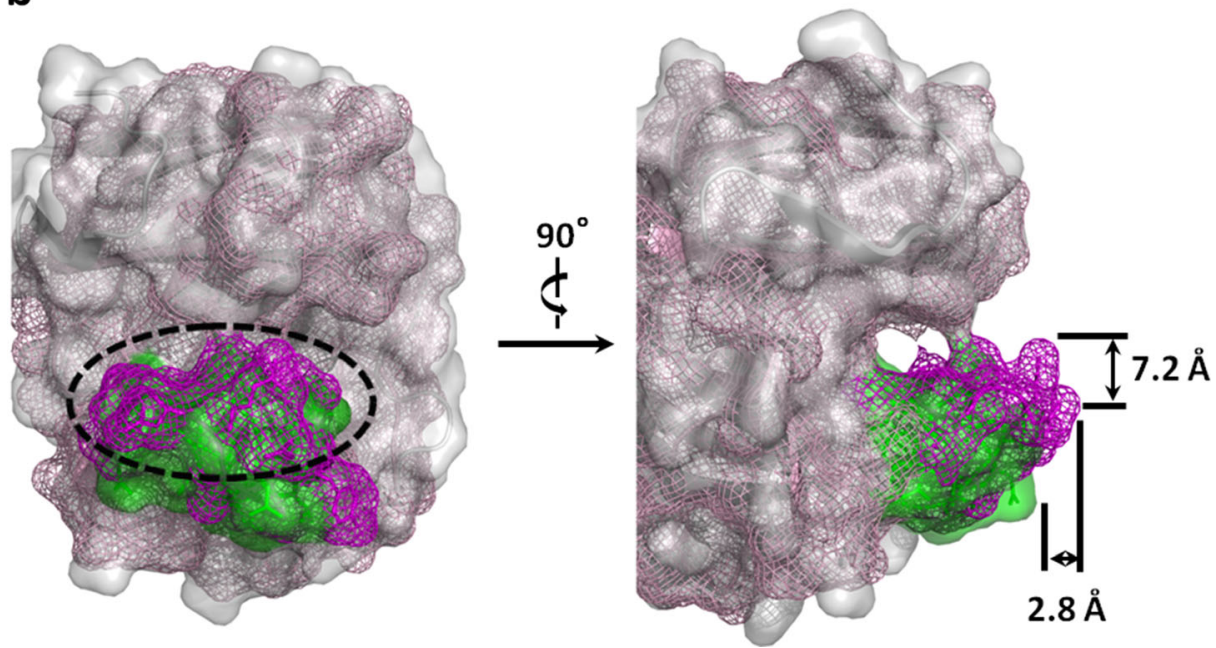

Fig. 8 Structure comparison of TcXylC $\Delta$ N17 and other xylanases. a Surface of the substrate-binding cleft of TcXylC $\Delta$ N17 drawn with wall-eyed stereo view in green. Stick models of inhibitors are colored gray (CtXyl) or yellow (NpXyl). b Superposition of TcXylC $\Delta \mathrm{N} 17$ and BcXyl viewed from the front and the side to represent the active site. The surfaces of TcXylC $\Delta \mathrm{N} 17$ and $\mathrm{BcXyl}$ are identified by color: gray (TcXylC $\Delta \mathrm{N} 17)$ and pale magenta (BcXyl). The "thumb" regions of TcXylC $\Delta \mathrm{N} 17$ and BcXyl are identified by color: green (TcXylC $\Delta \mathrm{N} 17)$ and magenta (BcXyl) (color figure online) 
$2.6 \AA$ apart from that in TcXylC $\Delta \mathrm{N} 17$. According to superposition of TcXylC $\Delta \mathrm{N} 17$ with $\mathrm{BcXyl}, \mathrm{TcXylC} \Delta \mathrm{N} 17$ is expected to be able to bind some branched sugar chain substrates more easily than BcXyl does.

\section{Conclusion}

From the genome database of Talaromyces cellulolyticus, seven kinds of ORF showing the homology to xylanase (GH11) were found. One of the recombinant enzymes, TcXylC, was crystallized, and the crystal structure was solved up to the resolution of $1.98 \AA$. In the asymmetric unit, two kinds of xylanase structures were observed: One shows an open form and the other shows a close form. This conformational change is induced in hydrogen bond networks between thumb and finger (Fig. 7c). Furthermore, because the values of $B$-factors of finger region in $A_{\text {close }}$ and thumb region in $B_{\text {open }}$ exhibit high values (Fig. $7 \mathrm{~d}$, e), these regions are able to fluctuate and are flexible. These results suggest that the tunnel has an open form in apo form as $B_{\text {open, }}$, and if the substrate binds to the active site cleft, the tunnel (close form) forms by replacing water molecules in $A_{\text {close }}$. The flexibility of the active site cleft plays a role in substrate binding and enzyme activity. From the structural comparison of xylanase, it was clarified that changing the flexible region between two different forms induces and accelerates the enzyme reaction.

Acknowledgments The X-ray diffraction data were obtained at the beamline BL44XU in SPring-8, Hyogo, Japan, with the approval of the Institute for Protein Research, Osaka University, Osaka, Japan (proposal no. 2012A6704).

Open Access This article is distributed under the terms of the Creative Commons Attribution License which permits any use, distribution, and reproduction in any medium, provided the original author(s) and the source are credited.

\section{References}

1. Törrönen, A., \& Rouvinen, J. (1995). Biochemistry, 34, 847-856.

2. Jänis, J., Hakanpää, J., Hakulinen, N., Ibatullin, F. M., Hoxha, A., Derrick, P. J., Rouvinen, J., \& Vainiotalo, P. (2005). FEBS Journal, 272, 2317-2333.

3. Vardakou, M., Dumon, C., Murray, J. W., Christakopoulos, P., Weiner, D. P., Juge, N., Lewis, R. J., Gilbert, H. J., \& Flint, J. E. (2008). Journal of Molecular Biology, 375, 1293-1305.

4. Wakarchuk, W. W., Robert, L., Campbell, R. L., Sung, W. L., Davoodi, J., \& Yaguchi, M. (1994). Protein Science, 3, 467-475.

5. Törrönen, A., Harkki, A., \& Rouvinen, J. (1994). The EMBO Journal, 13, 2493-2501.

6. Paës, G., Berrin, J. G., \& Beaugrand, J. (2012). Biotechnology Advances, 30, 562-592.

7. Goyal, A., Ghosh, B., \& Eveleigh, D. (1991). Bioresource Technology, 36, 37-50.

8. Wen, Z., Liao, W., \& Chen, S. (2005). Process Biochemistry, 40, 3087-3094.

9. Sehnem, N. T., Bittencourt, L. R., Camassola, M., \& Dillon, A. J. (2006). Applied Microbiology and Biotechnology, 72, 163-167.

10. Yamanobe, T., Mitsuishi, Y., \& Takasaki, Y. (1987). Agricultural and Biological Chemistry, 51, 65-74.

11. Fujii, T., Hoshino, T., Inoue, H., \& Yano, S. (2014). FEMS Microbiology Letters, 351, 32-41.

12. Fujii, T., Fang, X., Inoue, H., Murakami, K., \& Sawayama, S. (2009). Biotechnology for Biofuels, 2, 24.

13. Watanabe, M., Inoue, H., Inoue, B., Yoshimi, M., Fujii, T., \& Ishikawa, K. (2014). AMB Express, 4, 27.

14. Fang, X., Yano, S., Inoue, H., \& Sawayama, S. (2009). Journal of Bioscience and Bioengineering, 107, 256-261.

15. Gill, S. C., \& von Hippel, P. H. (1989). Analytical Biochemistry, 182, 319-326. 
16. Niesen, F. H., Berglund, H., \& Vedadi, M. (2007). Nature Protocols, 2, 2212-2221.

17. Otwinowski, Z., \& Minor, W. (1997). Methods in Enzymology, 276, 307-326.

18. Vagin, A., \& Teplyakov, A. (2010). Acta Crystallographica Section D: Biological Crystallography, 66, 22-25.

19. Emsley, P., \& Cowtan, K. (2004). Acta Crystallographica Section D: Biological Crystallography, 60, 21262132.

20. Murshudov, G. N., Skubák, P., Lebedev, A. A., Pannu, N. S., Steiner, R. A., Nicholls, R. A., Winn, M. D., Long, F., \& Vagin, A. A. (2011). Acta Crystallographica Section D: Biological Crystallography, 67, 355-367.

21. Laskowski, R. A., MacArthur, M. W., Moss, D. S., \& Thornton, J. M. (1993). Journal of Applied Crystallography, 26, 283-291.

22. Matthews, B. W. (1968). Journal of Molecular Biology, 33, 491-497. 